\title{
Pitch perception and retention: Two cumulative benefits of selective attention
}

\author{
LAURENT DEMANY, GASPARD MONTANDON, and CATHERINE SEMAL \\ CNRS and Université Victor Segalen, Bordeaux, France
}

\begin{abstract}
By presenting, before a "chord" of three pure tones with remote frequencies, a tone relatively close in frequency to one component ( $\mathrm{T} 1$ ) of the chord, one can direct the listener's attention onto T1 within the chord. In the first part of the present study, it was found that this increases the accuracy with which the pitch of T1 is perceived. The attentional cue improved the discrimination between the frequency of T1 and that of another tone (T2) presented immediately after the chord or very shortly (300 msec) after it. No improvement was found when T1 was presented alone instead of within a chord. A subsequent experiment, in which the chord and T2 were separated by either $300 \mathrm{msec}$ or $4 \mathrm{sec}$, indicated that the attentional cue improved not only the perception, but also the memorization of the pitch of T1 (especially when T1 was the intermediate component of the chord). It is argued that the positive effect of attention on memory took place when the pitch percept was encoded into memory, rather than after the formation of the pitch memory trace.
\end{abstract}

Normal listeners are able to focus their attention on a sentence produced by a given speaker while another speaker is also talking. Similarly, it is not difficult to attend selectively to a melody played on a flute while another melody is concurrently played on a cello, or vice versa. The research reported here was concerned with the effect of selective attention on the perception and the memorization of a basic attribute of periodic sounds - namely, their pitch.

Some previous studies have already suggested that attention can improve the perception of pitch, as well as other basic auditory attributes (Clément, 2001; Demany, Clément, \& Semal, 2001; Mondor \& Bregman, 1994; Spence \& Driver, 1994). In a speeded identification paradigm, the listeners tested by Spence and Driver (Experiment 6) had to identify as low $(345 \mathrm{~Hz})$ or high $(375 \mathrm{~Hz})$ the frequency (or pitch) of a target tone presented at random to the left or the right of midline by a loudspeaker. This target was preceded by an auditory spatial cue, which generally had the same position as the target and, thus, allowed the listeners to orient their attention appropriately in the spatial domain (without actually moving their heads). The frequency of the target was identified more quickly in that case than when the cue and the target had opposite positions. Analogous results were obtained by Mondor and Bregman, who used a duration identification task rather than a frequency identification task and who manipulated attention in the frequency/

This work was supported by a grant from the Centre National de la Recherche Scientifique (Programme Interdisciplinaire "Cognition et Traitement de l'Information"). The authors thank Nelson Cowan for fruitful discussions. Correspondence should be addressed to L. Demany, Laboratoire de Neurophysiologie, BP 63, Université Victor Segalen, 146 rue Leo Saignat, F-33076 Bordeaux Cedex, France (e-mail: laurent. demany@psyac.u-bordeaux2.fr).

Note-This article was accepted by the previous editorial team, headed by Neil Macmillan. pitch domain, rather than in the spatial domain. In contrast to Spence and Driver and to Mondor and Bregman, Demany et al. (2001) and Clément did not use a speeded identification paradigm; they measured, instead, perceptual sensitivity in two-interval discrimination tasks without time pressure. Demany et al. (2001) reported, in particular, that if an amplitude-modulated tone (T1) is preceded by a visual cue drawing attention to one of T1's characteristics (its carrier frequency, its intensity, or the speed of its modulation), the perception of a slight modification of this feature in a comparison tone $\mathrm{T} 2$ presented shortly thereafter is facilitated; the same modification appeared to be perceived less accurately when the visual cue was presented immediately after $\mathrm{T} 1$, rather than before $\mathrm{T} 1$.

In addition to improving the perception of a sound feature, can attention also improve its retention in memory? It is commonly assumed, instead, that "the decay of auditory sensory memory is ... unaffected by task demands in a silent poststimulus environment" (Cowan, Lichty, \& Grove, 1990, p. 267). According to this view, the retention of purely acoustic information is automatic, in contrast to the retention of verbal (i.e., categorical and abstract) information, which is strongly dependent on attention (Baddeley, 1986; Peterson \& Peterson, 1959). Such a view was supported in the study by Demany et al. (2001) that we mentioned above. In several experimental conditions, the two modulated tones to be compared, T1 and T2, were separated by a silent delay of at least $4 \mathrm{sec}$ - a delay for which discrimination was clearly limited by memory factors, rather than by sensory factors. The visual cue orienting attention was then presented either immediately after $\mathrm{T} 1$ or a little before T2. The discrimination of T1 and T2 was not better in the former case than in the latter case. So, although the cue appeared to affect the perception of T1 when it was presented before this stimulus, it failed to affect its retention when it was an immediate post-cue. Similar re- 
sults were obtained in a companion experiment in which $\mathrm{T} 1$ was replaced by a sum of three simultaneous tones, with different spatial localizations, and a visual cue drew the listeners' attention onto one component of this "chord": The visual cue appeared to increase the accuracy of pitch perception for the cued tone when it was presented before the chord, but it had no effect on the retention of this pitch when it was presented immediately after the chord (Clément, 2001, Experiment 4).

Before Demany et al. (2001) and Clément (2001), Massaro (1970) and Keller and Cowan (1994) had also reported results consistent with the idea that the retention of an auditory sensation - and more specifically, a pitch perceptis automatic. By contrast, however, Keller, Cowan, and Saults (1995) suggested that pitch retention can depend on attention. They claimed that memory for pitch can be improved by some covert rehearsal process, even in the absence of interfering sounds following the sound to be remembered. Pechmann and Mohr (1992) also drew this conclusion, although their data support it only for a group of musically naive listeners, and not for a group of amateur musicians. A problem is that these two studies may have been flawed by a methodological artifact. In both of them, on each trial, listeners had to compare the frequencies of two tones separated by some delay, and the authors assessed the effect of a distracting task performed during the delay on the discrimination of the two tones. A deleterious effect of the distracting task was supposed to imply that this task disrupted an efficient covert rehearsal process. Unfortunately, a quite different interpretation should be considered. During the distracting task, the listeners had to depress buttons, and this presumably produced audible sounds. The sounds in question were certainly weak. However, as has been shown by Semal and Demany (1993), even weak sounds can very significantly deteriorate the pitch memory trace generated by a previous tone that was much louder. Such interference phenomena are easily overlooked, and of course the mechanism of interference may be largely preattentional.

From the results of Massaro (1970), Keller and Cowan (1994), Demany et al. (2001), and Clément (2001), as well as those obtained with amateur musicians by Pechmann and Mohr (1992), one is led to believe that the retention of a pitch memory trace does not depend on attention after the formation of this trace. ${ }^{1}$ However, this does not mean that attention should play no role at all in pitch memory, because the formation of a pitch memory trace - or in other words, the encoding of a given pitch percept in memorymay, in itself, depend on attention. It is conceivable that a focusing of attention on the pitch of some sound while this sound is presented not only improves the perception of its pitch, but also improves, at the same time, the encoding of that pitch in memory and, in this way, the retention of its trace independently of the listener's mental activity during the retention delay. The present study was undertaken to test this hypothesis.

Six interrelated experiments were performed, among which four (Experiments 1, 3, 5, and 6) were conducted on the same group of listeners. Experiments $1-5$ were intended to confirm that attention can improve the accuracy of the pitch percept evoked by a tone, in a methodological framework which was similar to, but somewhat different from, that used by Demany et al. (2001) and Clément (2001). In Experiment 6, which was the main experiment, we wished to determine whether the perceptual benefit of attention demonstrated in Experiments 1-5 was restricted to perception per se or was associated with an additional benefit relating to memory.

\section{EXPERIMENTS 1 AND 2}

On each trial of these experiments, the listener was presented with a "chord" of three pure tones with distant frequencies. This chord was followed immediately or almost immediately by a single pure tone (T2), which was very slightly different in frequency (by $\pm \Delta F$ ) from a randomly selected component (T1) of the chord. The task was to judge whether $\mathrm{T} 2$ was higher or lower in frequency than $\mathrm{T} 1$, and we measured discrimination thresholds for $\Delta F$, using an adaptive procedure. In one condition, termed the cued condition, the chord was preceded by a tone orienting the listener's attention to $\mathrm{T} 1$. In another condition, termed the uncued condition, the chord was not preceded by any signal, so that the listener ignored the identity of T1 until the presentation of T2. By itself, T2 revealed unambiguously the identity of $\mathrm{T} 1$ since it was typically more than 30 times closer in frequency to T1 than to each of the other two components of the chord. We wished to know whether the $\Delta F$ thresholds would be lower in the cued condition than in the uncued condition. This outcome was predictable on the basis of the results reported by Demany et al. (2001) and Clément (2001).

\section{Experiment 1}

\section{Method}

Participants. Four students with normal hearing were tested in this experiment, as well as in Experiments 3, 5, and 6. Three of these listeners (E.B., G.M., and S.C.) were amateur musicians. One of them (G.M.) was the second author. G.M. had previously participated in other experiments on auditory perception and memory, but this was not the case for the other 3 listeners.

Stimuli. On each trial, the frequencies of the three simultaneous pure tones making up the chord were selected randomly, with a rectangular probability distribution on a logarithmic frequency scale extending from 250 to $4000 \mathrm{~Hz}$. However, each of them was constrained to be separated by a large and inharmonic musical interval from its neighbor(s). The interval in question was selected randomly between 1.08 and 1.32 octave (i.e., 1,296 and 1,584 musical cents). Thus, the lowest and highest components of the chord formed a musical interval that could vary from 2.16 octaves $(2,592$ cents) to 2.64 octaves $(3,168$ cents). The "relevant" component of the chord-that is, T1 - was equiprobably its lower component, its higher component, or the intermediate component. T2 was equiprobably higher or lower than $\mathrm{T} 1$ and followed the chord after a silent interstimulus time interval (ISI ${ }_{\mathrm{T} 1-\mathrm{T} 2}$ ) of $300 \mathrm{msec}$. In the cued condition, the chord was preceded by a pure tone that was positioned, at random, either 150 cents above T1 or 150 cents below T1; there was a silent ISI of $300 \mathrm{msec}$ between this cue and the chord. All tones had a total duration of $700 \mathrm{msec}$ and were gated on and off with 10 -msec cosinu- 
soidal amplitude ramps. They had a nominal sound pressure level of $60 \mathrm{~dB}$ and were presented binaurally (diotically) by means of Sennheiser HD265 headphones. They were generated at a sampling rate of $16000 \mathrm{~Hz}$, via a 16-bit digital-to-analog converter (Oros AU22) whose output was low-pass filtered at $6000 \mathrm{~Hz}$ (Stanford Research SR640)

Procedure. The listeners were tested individually in a doublewalled soundproof booth. They gave their responses (T2 higher than $T 1$ or $T 2$ lower than T1) by pressing one of two buttons on a response box. Feedback was provided visually: Following each correct response, a light-emitting diode (LED) located in front of the appropriate button was switched on for $300 \mathrm{msec}$; no LED was switched on if the response was wrong. In the cued condition as well as in the uncued condition, the onset of the chord presented on a given trial occurred 1,500 msec after the response given on the previous trial.

Thresholds for $\Delta F$ (expressed in cents rather than in Hertz) were measured using the weighted up-down adaptive procedure described and analyzed by Kaernbach (1991). During each block of trials, three thresholds were measured concurrently and independently: one threshold for each of the three possible positions of T1 within the chord. $\Delta F$ was initially set to the same value in the three adaptive tracks, well above the expected thresholds. Then, for each position of $\mathrm{T} 1, \Delta F$ was divided by $(1.5)^{0.25}$ after every correct response and was multiplied by 1.5 after every wrong response. A block terminated when, for each position of T1, at least 14 reversals had occurred in the variation of $\Delta F$. The median of the $\Delta F$ values used after the fourth of these 14 reversals was taken as the listener's threshold for the corresponding position of T1. For this $\Delta F$ value, the probability of a correct response could be estimated at .80 .

Within a given experimental session, four blocks of trials were run: two blocks in the cued condition and two blocks in the uncued condition. These two conditions were alternated from block to block, and the condition used on the first block changed from session to session. Seven sessions were run for each listener. The first three were considered as practice sessions, and the results reported below are those obtained during the four final sessions.

\section{Results and Discussion}

Figure 1 shows that, for each listener, the mean threshold measured in the cued condition was lower than the mean threshold measured in the uncued condition. On average, the presentation of cues reduced thresholds by about $28 \%$. This effect is comparable in size to the performance improvement previously observed in our laboratory for dichotic chords preceded by visuospatial cues (Clément, 2001, Experiment 4).

The 192 measured thresholds were submitted to a repeated measures analysis of variance (ANOVA) involving three factors: 2 cue conditions (cued vs. uncued) $\times 3$ positions of $\mathrm{T} 1$ within the chord (higher, lower, or intermediate) $\times 4$ listeners. The cue condition factor appeared to have a significant effect on thresholds $[F(1,3)=39.65$, $p=.008]$, as did the listener factor $[F(3,168)=9.11, p<$ $.001]$. This was not the case for the position of T1 factor $[F(2,6)=2.73, p=.14]$. The only significant interactions were the two-way interaction between the position of $\mathrm{T} 1$ and the listener factors $[F(6,168)=8.08, p<.001]$ and the three-way interaction $[F(6,168)=2.24, p=.042]$.

\section{Experiment 2}

\section{Rationale and Method}

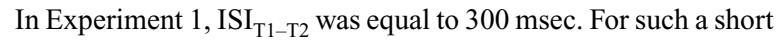
ISI, it seemed a priori reasonable to think that the internal noise limiting the discrimination of T1 and T2 would be restricted to sensory

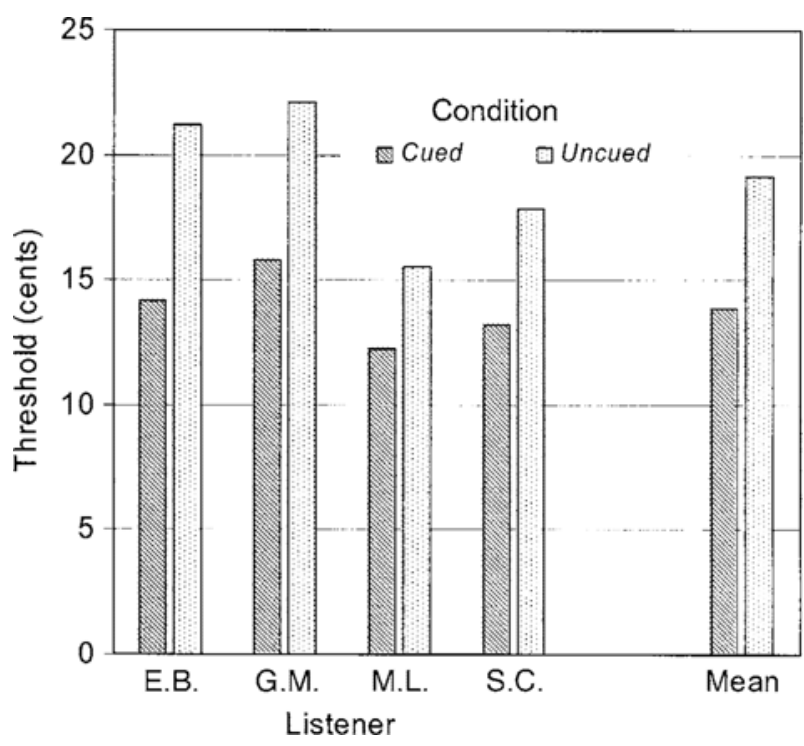

Figure 1. Results of Experiment 1: individual and grand means of the $\Delta F$ thresholds measured in the cued and uncued conditions. Thresholds were measured in musical cents: 1 cent $=1 / 100$ semitone $=1 / 1200$ octave; this unit thus corresponds to a frequency ratio of $2^{1 / 1200}$.

noise. Indeed, previous studies of frequency discrimination have suggested that a limitation of discrimination performance by memory noise begins to be detectable only for markedly longer ISIs (e.g., Harris, 1952). In these previous studies, however, participants had to compare isolated tones. In Experiment 1, by contrast, $\mathrm{T} 1$ was presented in the context of two distractor tones; therefore, part of the internal noise limiting discrimination performance may have been a memory noise. It was conceivable that the benefit of the cues consisted in a reduction of this memory noise, rather than the sensory noise. Consequently, we performed a revised version of Experiment 1 in which ISI $_{\mathrm{T} 1-\mathrm{T} 2}$ took two values: $300 \mathrm{msec}$, as before, and $0 \mathrm{msec}$. In the latter case, obviously, discrimination performance could not be limited by memory noise.

The 5 listeners who provided the data in this new experiment were again students with normal hearing. Each of them was an amateur musician. Only one of them (S.C.) had previously participated in Experiment 1 . Two additional listeners were dismissed following the initial training phase because they were apparently unable to carry out the task in any condition: Their thresholds always exceeded 200 cents. The experimental procedure was the same as before, except that, in each session, the two blocks of trials run in the cued condition and in the uncued condition now included one block with $\mathrm{ISI}_{\mathrm{T} 1-\mathrm{T} 2}=300 \mathrm{msec}$ and one block with $\mathrm{ISI}_{\mathrm{T} 1-\mathrm{T} 2}=0 \mathrm{msec}$. In the latter case, the 10-msec amplitude ramps located at the end of T1 and the beginning of $\mathrm{T} 2$ were sufficient to separate these two tones perceptually. The experiment proper consisted of eight sessionsthat is, eight threshold measurements in each of the four types of trial blocks.

\section{Results and Discussion}

The individual and mean results obtained in the four types of trial blocks are displayed in Table 1. For ISI T1-T2 $_{2}=$ $300 \mathrm{msec}$, the data were similar to those obtained in Experiment 1 . To our surprise, thresholds were lower for $\mathrm{ISI}_{\mathrm{T} 1-\mathrm{T} 2}=0 \mathrm{msec}$ than for ISI $\mathrm{I} 1-\mathrm{T} 2=300 \mathrm{msec}$; an ANOVA showed that the corresponding effect was reliable $[F(1,4)=$ 
Table 1

Individual and Grand Means of the $\Delta \boldsymbol{F}$ Thresholds Measured in the Cued and Uncued Conditions in Experiment 2, as a Function of ISI $_{\text {T1-T2 }}$

\begin{tabular}{cccccc}
\hline & \multicolumn{2}{c}{ ISI $_{\mathrm{T} 1-\mathrm{T} 2} 300 \mathrm{msec}$} & & \multicolumn{2}{c}{ ISI $_{\mathrm{T} 1-\mathrm{T} 2} 0 \mathrm{msec}$} \\
\cline { 2 - 3 } \cline { 5 - 6 } Listener & Cued & Uncued & & Cued & Uncued \\
\hline A.L. & 18.6 & 22.1 & & 15.7 & 17.6 \\
K.S. & 16.2 & 17.5 & & 12.6 & 14.4 \\
M.A. & 12.2 & 19.9 & & 8.3 & 10.2 \\
P.B. & 20.0 & 31.6 & & 19.6 & 24.0 \\
S.C. & 11.5 & 16.0 & & 8.3 & 10.8 \\
Mean & 15.7 & 21.4 & 12.9 & 15.4 \\
\hline
\end{tabular}

49.53, $p=.002]$. However, the presence of cues appeared to be significantly beneficial for each ISI $[300 \mathrm{msec}$, $F(1,4)=10.14, p=.033 ; 0 \mathrm{msec}, F(1,4)=24.59, p=$ $.008]$, and there was no significant interaction between the cue condition and the ISI factors $[F(1,4)=5.17, p=$ $.085]$. The fact that cuing was beneficial even in the absence of any delay between $\mathrm{T} 1$ and $\mathrm{T} 2$ supports the idea that attention can have a positive effect on the accuracy of pitch perception per se.

\section{EXPERIMENTS 3-5}

A natural interpretation of the results obtained in Experiments 1 and 2 is that the presence of a cue before a chord improved performance by orienting the listener's attention to $\mathrm{T} 1$ within the chord. However, this interpretation was not the only possible one. Alternative hypotheses were tested in three control experiments, Experiments 3, 4, and 5.

\section{Experiment 3}

It could be hypothesized that, in Experiments 1 and 2, the presentation of a cue improved the sensory encoding of T1 independently of attention or that the cue was efficient because, due to it, the listener knew more precisely when the chord would be presented and was thus better prepared for its presentation from a temporal point of view. We reasoned that if one or both of these suppositions were correct, one would expect to find that the discrimination between $\mathrm{T} 1$ and $\mathrm{T} 2$ is again better in the cued condition than in the uncued condition when $\mathrm{T} 1$ is presented alone instead of within a chord. On the basis of this reasoning, Experiment 3 was a replication of Experiment 1 without the distractor tones that had previously been presented together with $\mathrm{T} 1$.

One practice session and two test sessions were run. The test sessions were organized exactly like those in Experiment 1 , so that the collected data consisted of 24 threshold measurements for each of the 4 listeners. The individual and mean results obtained in the cued and the uncued conditions are displayed in Figure 2. An ANOVA revealed that the cue condition factor had a significant effect on thresholds $[F(1,3)=53.96, p=.006]$. However, as can be seen in Figure 2, thresholds were larger in the cued condition than in the uncued condition. The frequency regis- ter of $\mathrm{T} 1$ also had a significant effect $[F(2,6)=9.45, p=$ $.015]$, but there was no significant interaction.

The fact that the presentation of a cue had a deleterious effect on the discrimination between $\mathrm{T} 1$ and $\mathrm{T} 2$ provides evidence against the suppositions that we wished to test here. The exact source of this effect is unclear. Given that each cue was separated from the following T1 by a $300-\mathrm{msec}$ ISI, forward masking does not seem to be an appropriate explanation (Turner, Zeng, Relkin, \& Horwitz, 1992). One should think of a different proactive interference phenomenon (see, in this respect, Cowan, Saults, \& Nugent, 1997; Mondor \& Breau, 1999). In any case, it seems reasonable to hypothesize that the deleterious process involved here was also involved in Experiments 1 and 2, and reduced in these previous experiments the beneficial effect of the cues. More importantly, the present results make clear that the beneficial effect of the cues in Experiments 1 and 2 was crucially related to the presentation of distractor tones together with T1. The efficiency of these distractors is confirmed by the fact that, although the listeners tested in the present experiment were the same as those tested in Experiment 1 , thresholds were markedly lower here than in Experiment 1 (compare Figure 2 with Figure 1).

\section{Experiment 4}

If, in Experiments 1 and 2, the only virtue of the tonal cues was to serve as a general warning, orienting the listener's attention in the time domain but not in the frequency/pitch domain, an identical effect should be produced by bursts of wide-band noise. Experiment 4 tested this prediction. It was a replication of Experiment 1 with cues consisting of samples of pink noise. Like the tonal cues previously used, these new cues had an (average) intensity of $60 \mathrm{~dB}$ SPL,

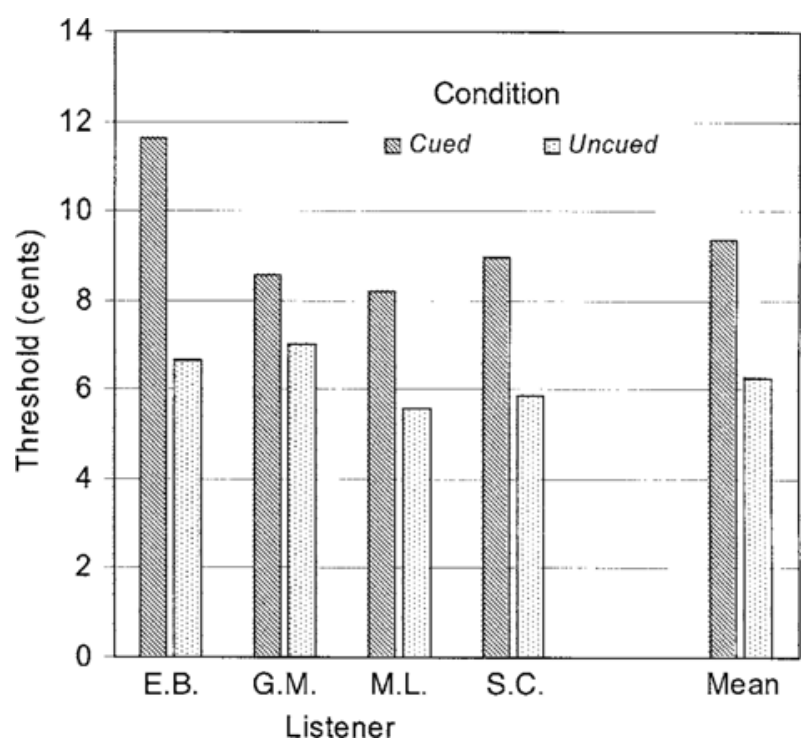

Figure 2. Results of Experiment 3: individual and grand means of the $\Delta F$ thresholds measured in the cued and uncued conditions. 
lasted $700 \mathrm{msec}$, and were separated from the chords by $300-\mathrm{msec}$ pauses. Five listeners were tested. Four of them had been tested before in Experiment 2, and the 5th listener belonged to the group tested in Experiments 1, 3, 5, and 6 .

In the cued condition, the individual means of the measured thresholds ranged from 12.0 to 26.9 cents, and the grand mean was 17.3 cents. In the uncued condition, the individual means ranged from 12.6 to 22.9 cents, and the grand mean was 16.6 cents. These two sets of thresholds did not differ significantly from each other $[F(1,4)<1]$. The absence of any advantage for the cued condition clearly rules out the hypothesis that, in Experiments 1 and 2, the cues were beneficial merely because they served as a general warning.

\section{Experiment 5}

When, in Experiments 1 and 2, a chord was preceded by a cue, this cue was presented at a distance of \pm 150 cents from $\mathrm{T} 1$. This choice resulted from a compromise. Of course, the cue had to be close to $\mathrm{T} 1$ in order to draw unambiguously the listener's attention to it within the chord. On the other hand, the cue had to differ from T1; otherwise, the listener could have profitably compared T2 with the cue itself, rather than with T1. But even for a cue that was at a distance of \pm 150 cents from $T 1$, it was still conceivable that the listener could ignore T1. A possible strategy was to compare the melodic interval formed by the cue and T2 (cue-T2) with an internal standard $(I)$ of approximately 150 cents and to adopt the following rules: (1) If cue-T2 is an ascending interval larger than $I$ or a descending interval smaller than $I$, respond that $\mathrm{T} 2$ is higher than $\mathrm{T} 1$; (2) if cue-T2 is an ascending interval smaller than $I$ or a descending interval larger than $I$, respond that $\mathrm{T} 2$ is lower than T1. In order to check that this strategy was not used in Experiment 1, we performed on the same listeners a modified version of its cued condition in which, on each trial, T1 was withdrawn from the chord and the task was to classify as large ( $>150$ cents) or small ( $<150$ cents) the cue-T2 interval. The underlying hypothesis was that, in Experiment 1 (or Experiment 2), the strategy described above would have been inefficient because the listeners were unable to assess the size of the $c u e-\mathrm{T} 2$ interval precisely enough.

Instead of again measuring thresholds, we assessed perceptual sensitivity in terms of $d^{\prime}$ for fixed frequency shifts $(\Delta F)$ between the virtual tone corresponding to T1 (withdrawn from the chord) and T2. For a given listener and position of T1 within the chord, the absolute value of $\Delta F(\mathrm{ex}-$ pressed in cents) was equal to the discrimination threshold measured in the cued condition of Experiment 1 for the same listener and position of T1. Therefore, if the listeners had used the strategy described above in Experiment 1, they should have been able here to respond correctly with a probability of .80 , and we should have obtained a mean $d^{\prime}$ of at least $1.68 .^{2}$ One practice session and one test session were run. The test session included four blocks of trials. Each block terminated when at least 50 trials had oc- curred for each of the three possible positions of T1. The results are easily summarized: $d^{\prime}$ never came close to 1.68 ; its grand mean did not even reach 0.10 . This outcome makes it clear that, in the cued condition of Experiment 1, the listeners based their judgments on the relation between $\mathrm{T} 2$ and $\mathrm{T} 1$, rather than on that between $\mathrm{T} 2$ and the cue.

\section{EXPERIMENT 6}

Experiments 1-5 were concerned with the influence of attention on pitch perception. In Experiment 6, by contrast, we searched for an influence of attention on pitch retention. The stimuli were essentially the same as those in Experiments 1 and 2, and we again compared data collected in the presence and the absence of a tonal cue before the chord presented on each trial. However, whereas ISI ${ }_{\mathrm{T} 1-\mathrm{T} 2}$ was always short (300 msec, at most) in Experiments 1 and 2, this interval was here either short again or much longer: Its two possible values were $300 \mathrm{msec}$ and $4 \mathrm{sec}$. Previous research has shown that the frequency discrimination of two pure tones is markedly poorer for an ISI of $4 \mathrm{sec}$ than for an ISI of $300 \mathrm{msec}$, especially when the frequencies of the tones vary widely from trial to trial (Clément, Demany, \& Semal, 1999; Harris, 1952). For a 4-sec ISI, it is clear that discrimination accuracy is limited much more by memory noise than by sensory noise. Therefore, if attention were able to affect pitch retention per se, this could be demonstrated here by an interaction between the cue condition factor (cued vs. uncued) and $\mathrm{ISI}_{\mathrm{T} 1-\mathrm{T} 2}$ : One would expect to find a smaller effect of ISI $_{\mathrm{T} 1-\mathrm{T} 2}$ on discrimination accuracy in the cued condition than in the uncued condition.

\section{Method}

The chords and the cues were selected exactly as in Experiment 1. However, this was not the case for T2, because as in Experiment 5, we assessed discrimination accuracy in terms of $d^{\prime}$ for fixed frequency shifts ( $\Delta F$, in cents) between $\mathrm{T} 1$ and $\mathrm{T} 2$. The value of $\Delta F$ used for a given listener, cue condition, and position of T1 within the chord corresponded to the mean frequency discrimination threshold measured in Experiment 1 for the same listener, cue condition, and position of T1. Thus, $\Delta F$ was smaller in the cued condition than in the uncued condition, and we expected to obtain the same $d^{\prime}$ values in these two conditions for $\mathrm{ISI}_{\mathrm{T} 1-\mathrm{T} 2}=300 \mathrm{msec}$.

The data were collected in 12 test sessions for each listener. ISI $_{\mathrm{T} 1-\mathrm{T} 2}$ was set to $300 \mathrm{msec}$ in 4 of them (Sessions 1, 4, 7, and 10) and to $4 \mathrm{sec}$ in the other 8 sessions. Each session included four blocks of trials: two blocks in the cued condition interleaved with two blocks in the uncued condition, as in Experiments 1 and 2. If ISI T1-T2 was equal to $300 \mathrm{msec}$, a block terminated when at least 50 trials had occurred for each of the three possible positions of T1 within the chord. If $\mathrm{ISI}_{\mathrm{T} 1-\mathrm{T} 2}$ was equal to $4 \mathrm{sec}$, the minimum number of trials required was 25 instead of 50 .

\section{Results and Discussion}

For a given listener, cue condition, position of $\mathrm{T} 1$ within the chord, and value of $\mathrm{ISI}_{\mathrm{T} 1-\mathrm{T} 2}$, four independent sets of at least 100 trials had been run (in single sessions for $\mathrm{ISI}_{\mathrm{T} 1-\mathrm{T} 2}=300 \mathrm{msec}$, and pairs of successive sessions for $\left.\mathrm{ISI}_{\mathrm{T} 1-\mathrm{T} 2}=4 \mathrm{sec}\right)$. A $d^{\prime}$ statistic was computed from each 
set, and the $192 d^{\prime}$ values thus obtained were submitted to an ANOVA. ${ }^{3}$ This ANOVA revealed, in addition to a significant main effect of ISI $_{\mathrm{T} 1-\mathrm{T} 2}[F(1,3)=95.50, p=$ .003 , a significant interaction between $\mathrm{ISI}_{\mathrm{T} 1-\mathrm{T} 2}$ and the cue condition factor $[F(1,3)=23.46, p=.017]$. These two effects are displayed in the left-hand panel of Figure 3. As was expected, for the shorter $\mathrm{ISI}_{\mathrm{T} 1-\mathrm{T} 2}, d^{\prime}$ took very similar average values in the cued and the uncued conditions. When ISI $\mathrm{T}_{\mathrm{T} 1 \mathrm{~T} 2}$ became long, $d^{\prime}$ decreased, but its decrease was smaller in the cued condition than in the uncued condition. The corresponding interaction implies that attention had a benefit on pitch retention. The ANOVA also revealed, however, the existence of two significant three-way interactions: (1) ISI $_{\mathrm{T} 1-\mathrm{T} 2} \times$ cue condition $\times$ position of T1 within the chord $[F(2,6)=5.81, p=.040]$ and (2) $\mathrm{ISI}_{\mathrm{T} 1-\mathrm{T} 2} \times$ cue condition $\times$ listener $[F(3,9)=$ $5.19, p=.024]$. The first of the three-way interactions is illustrated in the right-hand part of Figure 3. As can be seen, the advantage of the cued condition for pitch retention was much larger when T1 was the intermediate component of the chord than when T1 was either the lower or the higher component. This finding may have some relation with the fact that, in multivoiced (i.e., polyphonic) music, an inner voice is perceptually less salient than the highest voice or the lowest voice (Brochard, Drake, Botte \& McAdams, 1999; Huron \& Fantini, 1989; Palmer \& Holleran, 1994). In Experiment 1 or 2, however, the effect of cuing on pitch perception did not appear to be significantly dependent on the position of T1 within the chord. The second three-way interaction is illustrated in Figure 4, which shows that an advantage of the cued condition for pitch retention was observed in Listeners E.B., G.M., and S.C., but not in Listener M.L. In Experiment 1, M.L. was also the listener for whom cuing had the smallest effect on performance (Figure 1).

\section{GENERAL DISCUSSION}

Experiments 1-5 showed that selective attention can improve the precision of a pitch percept. This finding is consistent with the results of some previous studies, briefly reviewed in our introductory section (Clément, 2001; Demany et al., 2001; Spence \& Driver, 1994). From a more general point of view, the idea that the auditory system treats attended and ignored stimuli differently tallies with the literature concerning event-related potentials. In his review of the corresponding evidence, Hackley (1993) wrote: "Sensory processes are obligatory and invariant with
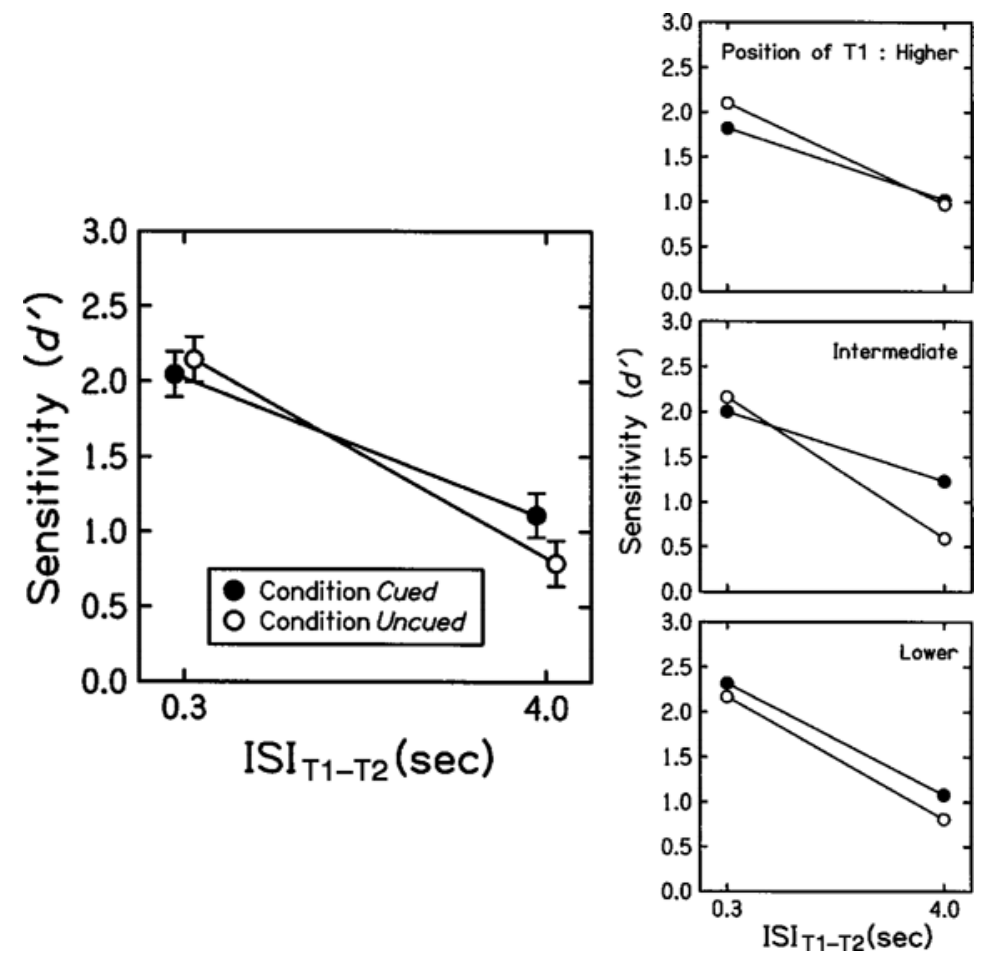

Figure 3. Results of Experiment 6. Left-hand panel: Mean $d^{\prime}$ values measured in the cued and uncued conditions as a function of ISI $_{\mathrm{T} 1-\mathrm{T} 2}$, the interstimulus interval between $\mathrm{T} 1$ and $\mathrm{T} 2$. Recall that $\Delta F$ was smaller in the cued condition than in the uncued condition. The error bars represent $95 \%$ confidence intervals, based on the error variance associated with the cue condition $\times$ ISI $_{\text {T1-T2 }}$ interaction (see Loftus \& Masson, 1994). In the three right-hand panels, the data are displayed as a function of the position of $\mathrm{T} 1$ within the chord. 


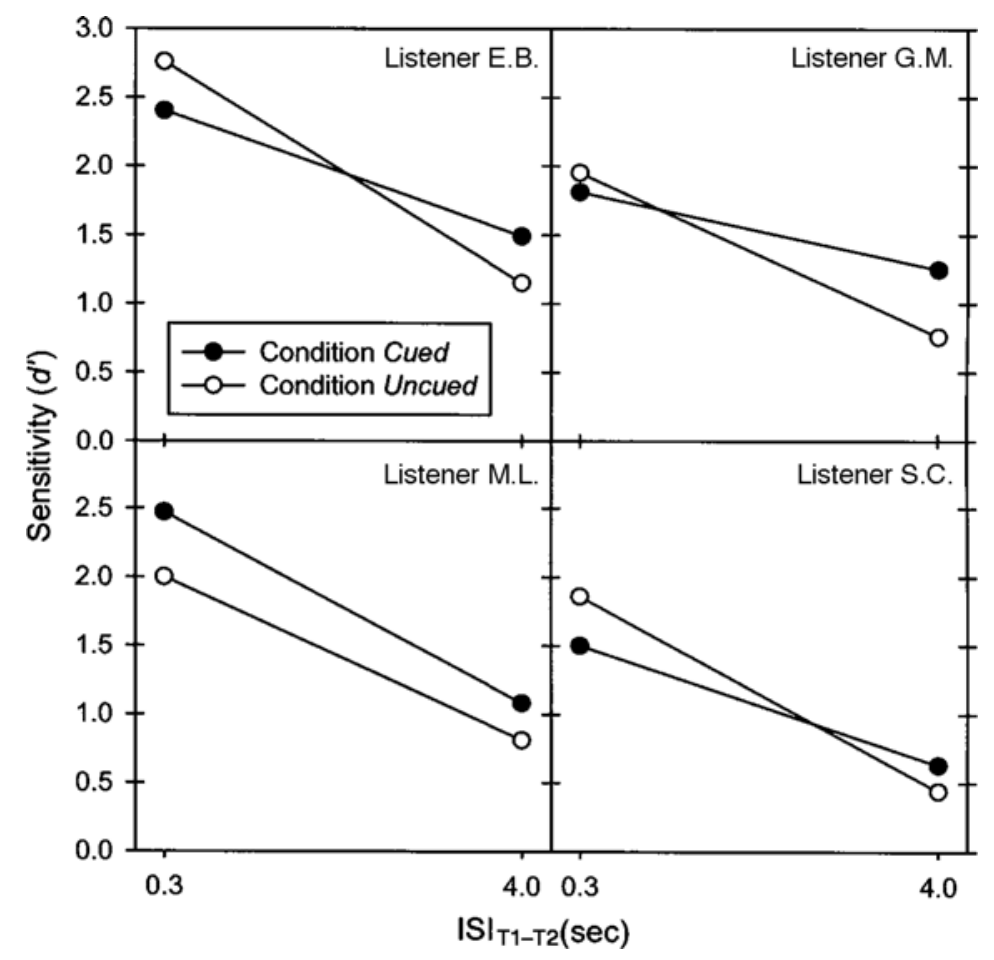

Figure 4. Individual results for the 4 listeners tested in Experiment 6.

attention at peripheral and brain-stem levels, [but] beginning at forebrain levels (i.e., thalamus or neocortex), there is a transition from full to partial automaticity. For the auditory modality, this transition begins immediately after the arrival of information at primary thalamo-recipient cortical areas" (p. 425). More recently, it has been claimed that selective attention can, in fact, modify auditory brainstem evoked potentials (Galbraith, Bhuta, Choate, Kitahara, \& Mullen, 1998; Galbraith \& Doan, 1995).

Experiment 6, however, indicated that the benefit of focusing attention on one tone while this tone is presented among other tones may not be limited to the perception of the focused tone's pitch. Instead, the perceptual benefit can be associated with a second benefit that relates to the retention in memory of the focused tone's pitch. Apparently, cumulative attentional effects of this kind had not been demonstrated up to now. As was mentioned in our introductory section, it was not clear from previous research that attention can affect pitch memory. We suggested, indeed, that the retention of a pitch memory trace does not depend on attention after the encoding of the pitch percept in memory. This means that a pitch memory trace cannot be successfully rehearsed, in contrast to verbal material. Nevertheless, attention might improve the retention of a pitch percept by affecting the encoding of the percept in memory. In other words, the positive effect of attention on retention in memory might take place when the pitch is perceived and its memory trace is formed, rather than fol- lowing the stimulus presentation. We believe that this is precisely what occurred in Experiment 6. In the domain of vision, an analogous suggestion was made by Tanaka and Sagi (2000). They found that a just-detectable Gabor signal briefly presented to one eye produces a monocular memory trace that is able to improve the detectability of a similar stimulus presented to the same eye several seconds later. This priming effect, however, appeared to depend on attention: The priming signal was most efficient when attention was drawn to it by a preceding temporal cue. The authors concluded that "attention affects transition between perception and memory."

A familiar notion is that attention can be directed to some perceptual entity in two ways: (1) reflexively, by exogenous mechanisms, or (2) voluntarily, by endogenous mechanisms (Laberge, 1995). In the present study, each cue presumably triggered exogenous attentional mechanisms that attracted attention in its frequency region. Given that the target (i.e., T1) was similar in frequency to the cue, one might suppose that attention to the target was attracted exogenously by the cue. On the other hand, an endogenous attentional process could also be used profitably, since each cue was "valid" (i.e., provided veracious information). Of these two potential sources of improvements in sensitivity, which was the dominant one? In this respect, it should be noted that, in vision as well as in audition, the facilitative exogenous effects of attentional cues on perception seem to be very rapid and transient phenom- 
ena, whereas endogenous attention works rather slowly and in a sustained manner (Mondor \& Breau, 1999; Müller \& Rabbitt, 1989; Spence \& Driver, 1994). In the present study, there was a rather long time interval $(1 \mathrm{sec})$ between the onset of a cue and the onset of the following chord. Moreover, informal observations suggested to us that the perceptual benefit of the cue did not decrease appreciably if this time interval was doubled by a lengthening of the silent ISI between the cue and the chord. Therefore, it is reasonable to think that the positive effects of attention observed here were based entirely on an endogenous process. Spence and Driver (1994, Experiments 3 and 7) found no effect of attention on the speed of pitch identifications in a situation in which attention was manipulated exogenously while an endogenous process was discouraged by making the cues equiprobably valid or invalid. By contrast, these authors found (in their Experiment 6) a positive effect of attention when both endogenous and exogenous mechanisms could be involved. They concluded that only endogenous attention can affect pitch perception. However, this conclusion seems to be inconsistent with the fact that, in their Experiment 6, they found a positive effect of attention even when the stimulus onset asynchrony between the cue and the target was as short as $100 \mathrm{msec}$.

Our main result being that attention can improve the retention of a pitch memory trace, probably by affecting the encoding of the pitch percept in memory, let us consider this result in the framework of a simple model of sensory memory, the diffusion model proposed by Kinchla and Smyzer (1967). ${ }^{4}$ According to this model, once a sensory value $\left(x_{0}\right)$ is encoded in memory, it goes through a random walk process: The value stored in memory is randomly increased or decreased by some constant amount at a constant rate. After a time $t$, consequently, this value differs from $x_{0}$ by some quantity that can be considered as a Gaussian random variable $\left(D_{t}\right)$ with a mean of 0 and a variance that is proportional to $t$ :

$$
\operatorname{Var}\left(D_{t}\right)=\Phi \cdot t,
$$

where $D_{t}$ represents memory noise and $\Phi$ can be referred to as its diffusion rate. When the task is, for instance, to make a relative judgment on the frequencies of two successive tones T1 and T2 separated by a time interval $t$, the subject's sensitivity will be limited by the sum of three independent internal noises: two sensory noises $\left(S_{\mathrm{T} 1}\right.$ and $S_{\mathrm{T} 2}$ ) representing the inaccuracies with which are perceived, respectively, $\mathrm{T} 1$ and $\mathrm{T} 2$, plus the memory noise representing the imperfect retention of T1. More precisely, the model states that, in terms of $d^{\prime}$, sensitivity will decrease with $t$ according to the equation

$$
d^{\prime}(t)=\frac{2 \delta}{\sqrt{\operatorname{Var}(S)+\Phi \cdot t}},
$$

in which $\delta$ is determined by the physical difference between $\mathrm{T} 1$ and $\mathrm{T} 2$ and $\operatorname{Var}(S)=\operatorname{Var}\left(S_{\mathrm{T} 1}\right)+\operatorname{Var}\left(S_{\mathrm{T} 2}\right)$.

If $t$ is small, sensitivity will be limited mostly by the overall sensory noise - that is, by $\operatorname{Var}(S)$. In Experiments 1 and 2, this was the case, and we found lower $\Delta F$ thresholds in the cued condition than in the uncued condition. We concluded that the presentation of an attentional cue before the chord reduced $\operatorname{Var}(S)$ [mostly $\operatorname{Var}\left(S_{\mathrm{T} 1}\right)$, presumably]. Consider, then, the expected effect of an attentional cue on the variation of $d^{\prime}$ as $t$ is increased, under the assumption that the cue reduces $\operatorname{Var}(S)$ but does not affect $\Phi$, the diffusion rate of the memory noise. Equation $2 \mathrm{im}-$ plies that $d^{\prime}$ should decrease more rapidly in the presence of the cue than in its absence. What we observed in Experiment 6 was just the opposite. In the framework of the model, this outcome implies, first of all, that the presentation of a cue reduced $\Phi$. More interestingly, however, the model also suggests that, in Experiment 6, the real effect of attention on the memory decay per se (i.e., $\Phi$ ) was larger than the effect empirically measured in terms of changes in $d^{\prime}$. From the mean data obtained for each of the three positions of $\mathrm{T} 1$ within the chords (Figure 3, right-hand panels), we estimated, using Equation 2, the ratio of the values taken by $\Phi$ in the cued and the uncued conditions $\left(\Phi_{\text {Uncued }} / \Phi_{\text {Cued }}\right)$. In doing so, we assumed that, in Equation 2 , for a given T1, $\delta$ is proportional to the frequency distance of T1 and T2 (in cents or in Hertz; these two units are almost equivalent if $\mathrm{T} 2$ is only slightly different from T1). This assumption, which was justified by previous data on the shape of the psychometric function in frequency discrimination tasks (e.g., Nelson \& Freyman, 1986), meant that the ratio of the values taken by $\delta$ in the cued and the uncued conditions was equal to the ratio of the frequency shifts made in these two conditions. The obtained estimates of $\Phi_{\text {Uncued }} / \Phi_{\text {Cued }}$ were 3.11, 14.74, and 2.42 for the lower, intermediate, and higher position of $\mathrm{T} 1$ within the chords, respectively. Thus, $\Phi_{\text {Uncued }}$ appeared to be markedly larger than $\Phi_{\text {Cued }}$ even for the lower and higher positions of T1. However, with respect to the relative values of $\operatorname{Var}(S)$ in the cued and the uncued conditions, the model led to an anomalous conclusion. For the lower and higher positions of T1, $\operatorname{Var}(S)_{\text {Uncued }} / \operatorname{Var}(S)_{\text {Cued }}$ had theoretical values (1.29 and 1.31, respectively) that were consistent with the results of Experiment 2. But for the intermediate position, $\operatorname{Var}(S)_{\text {Uncued }} / \operatorname{Var}(S)_{\text {Cued }}$ was in theory as low as $0.01 .^{5}$ This aberrant outcome of the model is liable to stem merely from statistical noise in our measurements of $d^{\prime}$. Nonetheless, let us point out that the model itself may be basically wrong. We have actually challenged it in another study on pitch memory, not concerned with attention (Demany, Montandon, \& Semal, 2004).

\section{REFERENCES}

BADDELEY, A. D. (1986). Working memory. Oxford: Oxford University Press, Clarendon Press.

Braida, L. D., \& Durlach, N. I. (1988). Peripheral and central factors in intensity perception. In G. M. Edelman, W. E. Gall, \& W. M. Cowan (Eds.), Auditory function (pp. 559-583). New York: Wiley.

Brochard, R., Drake, C., Botte, M. C., \& McAdams, S. (1999). Perceptual organization of complex auditory sequences: Effect of number of simultaneous subsequences and frequency separation. Journal of Experimental Psychology: Human Perception \& Performance, 25, $1742-1759$. 
CLÉMENT, S. (2001). La mémoire auditive humaine: Psychophysique et neuroimagerie fonctionnelle. Unpublished doctoral thesis, Université Victor Segalen, Bordeaux, France.

Clément, S., Demany, L., \& Semal, C. (1999). Memory for pitch versus memory for loudness. Journal of the Acoustical Society of America, 106, 2805-2811.

Cowan, N., Lichty, W., \& Grove, T. R. (1990). Properties of memory for unattended spoken syllables. Journal of Experimental Psychology: Learning, Memory, \& Cognition, 16, 258-269.

Cowan, N., Saults, J. S., \& NugENT, L. D. (1997). The role of absolute and relative amounts of time in forgetting within immediate memory: The case of tone-pitch comparisons. Psychonomic Bulletin \& Review, 4, 393-397.

Demany, L., Clément, S., \& Semal, C. (2001). Does auditory memory depend on attention? In D. J. Breebaart, A. J. M. Houtsma, A. Kohlrausch, V. F. Prijs, \& R. Schoonhoven (Eds.), Physiological and psychophysical bases of auditory function (pp. 461-467). Maastricht, The Netherlands: Shaker.

Demany, L., Montandon, G., \& Semal, C. (2004). Internal noise and memory for pitch. In D. Pressnitzer, A. de Cheveigné, S. McAdams, \& L. Collet (Eds.), Auditory signal processing: Physiology, psychoacoustics, and models (pp. 136-144). New York: Springer-Verlag.

Durlach, N. I., \& Braida, L. D. (1969). Intensity perception: I. Preliminary theory of intensity resolution. Journal of the Acoustical Society of America, 46, 372-383.

Galbraith, G. C., BhUta, S. M., Choate, A. K., Kitahara, J. M., \& Mullen, T. A. (1998). Brain stem frequency-following response to dichotic vowels during attention. NeuroReport, 9, 1889-1893.

Galbraith, G. C., \& Doan, B. Q. (1995). Brainstem frequencyfollowing and behavioral responses during selective attention to pure tone and missing fundamental stimuli. International Journal of Psychophysiology, 19, 203-214.

HACKLEY, S. A. (1993). An evaluation of the automaticity of sensory processing using event-related potentials and brain-stem reflexes. Psychophysiology, 30, 415-428.

HARRIS, J. D. $(1952)$. The decline of pitch discrimination with time. Journal of Experimental Psychology, 43, 96-99.

HURON, D., \& FANTINI, D. (1989). The avoidance of inner-voice entries: Perceptual evidence and musical practice. Music Perception, 9, 93-104.

KAERnBaCH, C. (1991). Simple adaptive testing with the weighted up-down method. Perception \& Psychophysics, 49, 227-229.

Keller, T. A., \& Cowan, N. (1994). Developmental increase in the duration of memory for tone pitch. Developmental Psychology, 30, 855-863.

Keller, T. A., Cowan, N., \& SAUlTS, J. S. (1995). Can auditory memory for tone pitch be rehearsed? Journal of Experimental Psychology: Learning, Memory, \& Cognition, 21, 635-645.

KINCHLA, R. A., \& SMYZER, F. (1967). A diffusion model of perceptual memory. Perception \& Psychophysics, 2, 219-229.

LABERGE, D. (1995). Attentional processing. Cambridge, MA: Harvard University Press.

Loftus, G. R., \& MAsson, M. E. J. (1994). Using confidence intervals in within-subjects designs. Psychonomic Bulletin \& Review, 1, 476-490.

Macmillan, N. A., \& Creelman, C. D. (1991). Detection theory: A user's guide. Cambridge: Cambridge University Press.

Massaro, D. W. (1970). Retroactive interference in short-term recognition memory for pitch. Journal of Experimental Psychology, 83, 32-39.

Mondor, T. A., \& Breau, L. M. (1999). Facilitative and inhibitory effects of location and frequency cues: Evidence of a modulation in perceptual sensitivity. Perception \& Psychophysics, 61, 438-444.

Mondor, T. A., \& Bregman, A. S. (1994). Allocating attention to frequency regions. Perception \& Psychophysics, 56, 268-276.

Müller, H. J., \& RABbitT, P. M. A. (1989). Reflexive and voluntary orienting of visual attention: Time course of activation and resistance to interruption. Journal of Experimental Psychology: Human Perception \& Performance, 15, 315-330.

NeLson, D. A., \& Freyman, R. L. (1986). Psychometric functions for frequency discrimination from listeners with sensorineural hearing loss. Journal of the Acoustical Society of America, 79, 799-805.

Palmer, C., \& Holleran, S. (1994). Harmonic, melodic, and frequency height influences in the perception of multivoiced music. Perception \& Psychophysics, 56, 301-312.

PechmanN, T., \& MOHR, G. (1992). Interference in memory for tonal pitch: Implications for a working-memory model. Memory \& Cognition, 20, 314-320.

Peterson, L. R., \& Peterson, M. J. (1959). Short-term retention of individual verbal items. Journal of Experimental Psychology, 58, 193198.

Semal, C., \& Demany, L. (1993). Further evidence for an autonomous processing of pitch in auditory short-term memory. Journal of the Acoustical Society of America, 94, 1315-1322.

SPENCE, C. J., \& DRIVER, J. (1994). Covert spatial orienting in audition: Exogenous and endogenous mechanisms. Journal of Experimental Psychology: Human Perception \& Performance, 20, 555-574.

TANAKA, Y., \& SAGI, D. (2000). Attention and short-term memory in contrast detection. Vision Research, 40, 1089-1100.

Turner, C. W., Zeng, F. G., RelKin, E. M., \& Horwitz, A. R. (1992). Frequency discrimination in forward and backward masking. Journal of the Acoustical Society of America, 92, 3102-3108.

\section{NOTES}

1. According to the results of Keller and Cowan (1994), this would be true even for retention delays exceeding $10 \mathrm{sec}$.

2. The expected value of $d^{\prime}$ was equal to 1.68 if, in Experiment 1 as well as the present experiment, response biases were completely absent. In the presence of response biases, $d^{\prime}$ should have been even larger.

3 . In 3 cases out of $192, d^{\prime}$ was infinite and had to be made finite artificially. We used the $1 / 2 N$ correction procedure described by Macmillan and Creelman (1991, p. 10).

4. This model has been subsequently incorporated by Durlach and Braida (1969; see also Braida \& Durlach, 1988) into a more general theoretical framework, taking into account context-coding processes in discrimination tasks.

5. According to the model, $\operatorname{Var}(S)_{\text {Uncued }}$ was extremely low for the intermediate position of 11 (much lower than for the other two possible positions of T1). It follows from Equation 2 that if $\operatorname{Var}(S)$ is equal to zero, whatever the value of $\Phi$, the $d^{\prime}$ value obtained for ISI $_{\mathrm{T} 1-\mathrm{T} 2}=0.3 \mathrm{sec}$ $\left[d^{\prime}(0.3)\right]$ should be 3.65 times larger than the $d^{\prime}$ value obtained for $\mathrm{ISI}_{\mathrm{T} 1-\mathrm{T} 2}=4 \mathrm{sec}\left[d^{\prime}(4)\right]$. For the uncued condition and the intermediate position of T1, the two $d^{\prime}$ values plotted in Figure 3 differ by a factor of 3.64 !

(Manuscript received July 11, 2002;

revision accepted for publication October 2, 2003.) 